\title{
Early versus delayed repair of vesicouterine fistula
}

\author{
Mathieu Bettez, MD; Guy Breault, MD; ${ }^{+}$Lesley Carr, MD MSc, ${ }^{ \pm}$Le Mai Tu, MD MSc
}

*The Urology Division, Department of Surgery, University of Sherbrooke, Sherbrooke, QC; †The Urology Division, Department of Surgery, University of Moncton, Hôpital Régional Georges L. Dumont, Moncton, NB; : The Urology Division, Department of Urogynecology, University of Toronto, Sunnybrook Health Sciences Centre, Toronto, ON

Cite as: Can Urol Assoc J 2011;5(2):e52-e55; D01:10.5489/cuaj.10065

\begin{abstract}
Vesicouterine fistulas (VUF) are a pathological and uncommon connection between the uterus and the bladder. Although rare, they are usually related to cesarean section. Nowadays, their incidence increases because of the increase in Cesarean sections. Patients with VUF may have various clinical presentations. The main symptoms are urinary incontinence, which may be associated with hematuria. Vesicouterine fistulas are usually associated with psychological distress and have a negative effect on quality of life. The accurate and early diagnosis of VUF can be difficult. There are multiple ways to investigate VUF and several examinations may be required to confirm the diagnosis; these examinations may include a cystoscopy, a retrograde cystography, methylene blue test and a computed tomography scan. We present 3 cases of vesicouterine fistula with successful surgical repair in which 2 patients had an early repair. One patient had an early surgical repair because of intractable pain and the other patient because of the large size of the fistula. The fistula repair surgeries were uneventful and the patients had an unremarkable recovery. We report that early surgical management is technically feasible without significantly increasing the difficulty of the surgery, with an excellent outcome in selected patients.
\end{abstract}

\section{Introduction}

Vesicouterine fistulas (VUF) are the least common type of urogenital fistulas, which is defined as an uncommon communication between the bladder and the uterus or the cervix. ${ }^{1}$ It may have an early or delayed presentation. Patients usually experience urinary incontinence (intermittent or continuous) which may be associated with hematuria. ${ }^{2}$ The diagnosis is usually made with cystoscopy, cystography and/ or computed tomography (CT) scan. ${ }^{3}$ It is usually recommended to delay surgical repair. ${ }^{4,5}$ However, recent cases demonstrate successful fistula closure with conservative management. ${ }^{3,6}$ We report 3 cases of successful surgical repair of VUFs. Two of these patients had an early repair.

\section{Case 1}

A 38-year-old female, G5P3, was admitted at 39 weeks of gestation for an elective Cesarean section because she had 3 previous Cesarean sections. During the surgery, the bladder was very adherent to the uterus and was close to the hysterotomy. At the end of the procedure, macroscopic hematuria was documented and resolved spontaneously. The patient was readmitted 17 days after surgery for intermittent hematuria and progressive abdominal pain exacerbated by voiding. An abdominal and pelvic ultrasound revealed a normal bladder and a small quantity of fluid in the uterine cavity. A cystoscopy showed an inflamed area on the right posterolateral wall of the bladder, with bleeding from a fistulous track. A retrograde cystography demonstrated a VUF (Fig.1). After a discussion with the patient, an immediate surgical repair was chosen.

During the fistula repair, the inflammation was impressive. Direct observation revealed 2 fistulas between the posterior wall of the bladder and the anterior wall of the uterus. The fistulous tracks were excised with a concurrent subtotal hysterectomy and an omental flap interposition. The patient recovered completely and did not have further hematuria or abdominal pain.

\section{Case 2}

A 31-year-old pregnant woman, G2P1, was admitted at term in spontaneous labour. Her past obstetric history was significant for a previous Cesarean section due to an arrest of the active phase of labour. The patient desired a trial of labour and was counselled about vaginal birth after Cesarean section. As the fetal head was descending, the patient felt intense abdominal pain and there were late decelerations noted on the fetal heart tracing. Simpson's forceps were used for delivery with mediolateral episiotomy. She delivered a live born female (3615 g).

The postpartum course was significant for gross hematuria and abdominal pain. As a small amount of urine may 


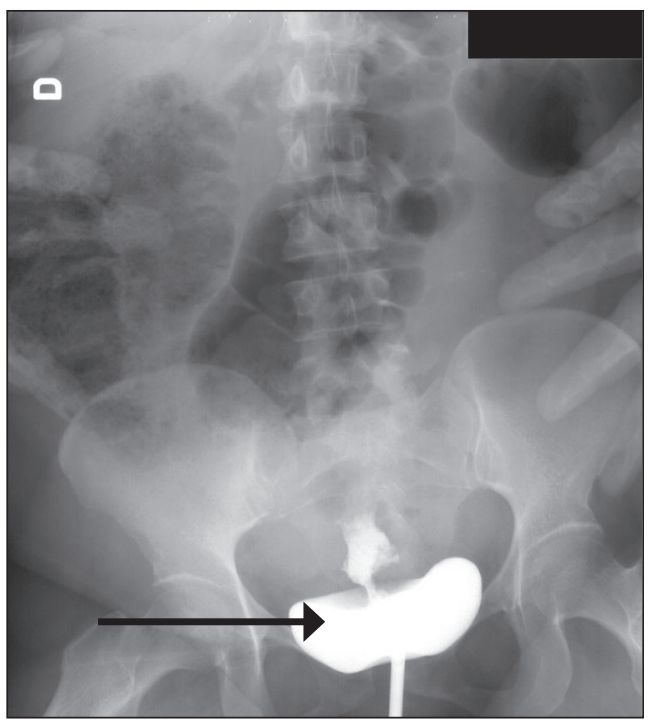

Fig. 1. A retrograde cystography showing the vesicouterine fistula.

have leaked around the catheter, it was changed from $12 \mathrm{~F}$ to $16 \mathrm{~F}$ by the gynecology service. Methylene blue test was performed due to continuous leakage; it was positive for VUF. A cystourethrography and a pelvic CT scan were performed which revealed a large vesicouterine fistula. Due to the large size of the fistula, early recognition and pain, immediate surgical repair was opted.

The surgery was done 3 days after delivery. There was a 5 -cm long fistula between the uterus and the intertrigonal ridge that was dissected and the uterus and the bladder were closed primarily with an omental flap interposition. The recovery was uneventful with no recurrence of symptoms.

\section{Case 3}

A 36-year-old woman, G2P2, consulted for a 10-month history of continuous urinary incontinence that started 1 month after her last Cesarean section. She also complained of some degree of stress and urge urinary incontinence. The patient did not report cyclic menouria. Her past obstetric history was significant for 2 prior Cesarean sections.

The workup included an abdominal, pelvic and transvaginal ultrasound which were negative. Retrograde cystography was also unremarkable. The initial cystoscopy examination was suboptimal because the patient experienced a lot of pelvic spasms. A second cystoscopy and a vaginal examination were performed under general anesthesia and revealed a moderate size fistula above the left trigone. The fistulous tract was further explored with a flexible ureteroscope, which finally confirmed a VUF.

The surgical repair included a fistulotomy with sharp dissection of the plane between the uterus, the bladder and the vagina. A small laceration was made on the uterus, but was closed primarily. An omental flap was placed between the uterine repair and the bladder closure. She never experienced a recurrence of urinary leakage.

\section{Discussion}

Vesicouterine fistulas are an uncommon and pathological communication between the bladder and the uterus or the cervix and represent $1 \%$ to $4 \%$ of all urogenital fistulas, with a peak incidence in young women between 25 and 33 years old. 4,7,8 The exact epidemiology is not well known. The rise in prevalence may be explained by the increases in Cesarean section and increases in vaginal delivery following previous Cesarean section.

The main causes of urogenital fistulas can be classified as obstetrical, surgical, radiation necrosis or related to a malignancy. Currently, the main cause of VUF is an iatrogenic injury during Cesarean section which accounts for $83-88 \%$ of cases. ${ }^{4,7}$ These injuries occur 2 times more often after repeated Cesarean sections. ${ }^{7,8}$ Some risk factors have been advocated for the development of VUF, such as an inadequate reflection of the bladder from lower uterine segment, excessive intra-operative bleeding, severe dystocia, forceps delivery, obstetrical vacuum, manual removal of the placenta, placenta percreta, uterine rupture, previous Cesarean section and dilatation and curettage. Other less frequent causes are inflammatory bowel diseases, endometriosis, intrauterine device migration, bladder tuberculosis and congenital lesions. ${ }^{2,9}$

Repeated Cesarean sections may result in progressive devitalization and scarring of the uterus and bladder base by damaging their vascular network, thus predisposing to fistula formation. ${ }^{4,7}$ In case 2 , a traumatic uterine rupture leading to the fistula probably developed when the patient started to have pain during labour. This laceration can be explained by the thinning of the lower base of the uterus and the presence of scar tissue related to the previous Cesarean sections. When the uterus ruptures, shearing force may be transmitted to the bladder thereby causing the fistula.

Patients with VUF can have various clinical presentations. Usually, immediate presentation occurs when there is direct injury to the bladder during surgery. Patients can have early hematuria and/or urinary leakage (as in cases 1 and 2), voiding difficulty, low-grade pyrexia, urinary sepsis or be completely asymptomatic. ${ }^{9}$ Delayed presentation can occur when there is an infection or a progressive devitalization of the posterior wall of the bladder. ${ }^{4,8}$ Patients with delayed presentation often have symptoms of urinary leakage from the vagina if the cervix is incompetent, cyclic hematuria (menouria), amenorrhea, infertility or first trimester abortion., ${ }^{4,8}$

Accurate and early diagnosis of vesicouterine fistula can be difficult, since there are many different clinical pictures. There are multiple means of investigation for VUF and sev- 
Bettez et al.

eral examinations may be required to confirm the diagnostic. When there is macroscopic hematuria and/or urinary leakage early in the postoperative period of Cesarean section or other gynecological procedures, we recommend early exclusion of VUF. ${ }^{2}$ The mainstays of diagnostic are cystoscopy and urinary tract imaging. ${ }^{2}$ A cystoscopy may be necessary to detect a fistula tract and to evaluate location, size and proximity of ureteral orifices. ${ }^{2}$ The diagnosis of VUF can be made indirectly by injection of methylene blue in the bladder. A VUF is present if dye comes out of the uterine cervix. Radiological evaluation remains the gold standard evaluation for diagnosis of urogenital fistulas. The integrity of the upper urinary tract should also be evaluated with an intravenous pyelography, a retrograde pyelography or a pyridium test. ${ }^{10} \mathrm{~A}$ cystography may demonstrate a tract or retrograde filling of the uterine cavity. During hysterography, if a VUF is present, the radio-opaque solution fills the bladder. Additional diagnostic procedures include contrastenhanced CT, MRI and vaginal ultrasound with or without Doppler (abnormal fluid in the uterine cavity). ${ }^{1,9}$

Treatment options for VUF include conservative, medical or surgical treatment. ${ }^{4,8,11,12}$ Some studies advocate conservative treatment for early diagnosed small fistula with bladder catheterization alone with antibiotics for 3 weeks. ${ }^{2}$ Others proposed induced amenorrhea by oral contraceptive pills or by luteinizing hormone-releasing hormone analog because epithelial and stromal cells that contain sex hormone receptors (as in the endometrium) had been observed within the fistula. ${ }^{1}$ This management technique may be less successful in women with a mature tract ( 6 weeks or longer). The ideal length of treatment is unknown, but most reports treated the patient for 6 months. ${ }^{2}$ There are also some reports describing the endoscopic fulguration of the fistula. ${ }^{10}$ Spontaneous closure of the fistula in up to $5 \%$ of cases may be expected with the involution of the uterus.

Surgery is the treatment of choice in most cases, especially for large vesicouterine fistulas which invariably necessitate surgical closure. ${ }^{4,10}$ Hysterectomy is not always necessary. The first effort at surgical closure of the fistula is the most likely to succeed. Surgical repair of VUF are performed by different approaches, which include vaginal, transvesical, transperitoneal and laparoscopic or robotic procedures. ${ }^{13}$ A bladder injury diagnosed during the Cesarean section should be repaired preferably with tissue interposition during the same surgery to prevent fistula development and the need for repeat surgeries in an already fibrous environment. When there is urinary leakage and pain related to VUF, early diagnosis and treatment are warranted to avoid social and familial embarrassment. ${ }^{14}$ It is usually recommended to delay surgery up to 3 months after the causative surgery to allow spontaneous closure of the fistula with the involution of the uterus and to have less inflammation making the surgery easier to perform with a lower risk of complica- tion. ${ }^{4,5}$ However, if it is suspected that the fistula is large or the patient has pronounced abdominal pain (as in cases 1 and 2) then immediate repair may be preferred to expedite recovery and minimize impact on quality of life during a period of expectant management.

In our first 2 cases, the surgeries were done early after delivery. In the first case, this timing was opted because the patient was experiencing significant abdominal pain. In this case, the inflammation and adhesions from previous surgeries were very important, forcing us to perform a subtotal hysterectomy. The patient did not wish to have further pregnancies. In the second case, early treatment was preferred over delayed surgery because of the large size of the fistula, which was not expected to resolve spontaneously, and because of the social and psychological concern of the disease. These 2 cases were associated with a good therapeutic success even though the procedures were slightly more difficult and one of the cases was associated with a subtotal hysterectomy.

In contrast to what is currently established in the literature, we recommend early surgical treatment of VUF for selected patients with urinary leakage and/or important discomfort, even though there is a 5\% chance of spontaneous closure of the fistula.

Despite the increase in incidence, this pathology remains rare. Due to this fact, our experience can only be presented as a case series. This type of report contains certain limitations. We cannot compare our technique to late repair or conservative management. All the publications on this topic are on a small number of patients due to the low incidence of VUF. Our goal is to offer patients all possible options and to try to render them more satisfied regarding the potential implication of VUF. The subjects for earlier repair of VUF must be chosen properly and patients should be offered all the possible treatment options.

\section{Conclusion}

Vesicouterine fistulas are uncommon, yet they are becoming more prevalent due to changes in modern obstetrical care. They should always be top-of-mind in patients with a history of Cesarean section or who have experienced a gynecological procedure associated with signs of hematuria and/or urinary leakage. The cases reported in this paper outline the varying clinical presentation and potential success of early surgical treatment of VUF in selected patients.

Acknowledgements: Special thanks to Tania Fayad and Patrick Richard.

Competing interests: None declared. 
Early versus delayed repair of vesicouterine fistula

This paper has been peer-reviewed.

\section{References}

1. Hadzi-Diokic JB, Pejici TP, Colovic VC. Vesico-uterine fistula: report of 14 cases. BJU Int 2007;100:1361-3.

2. DiMarco CS, DiMarco DS, Klingele CJ, et al. Vesicouterine fistula: a review of eight cases. Int Urogynecol J Pelvic Floor Dysfunct 2006; 17:395-9.

3. Yokoyama M, Arisawa C, Ando M. Successful management of vesicouterine fistula by luteinizing hormonereleasing hormone analog. Int I Urol 2006;13:457-9.

4. Porcaro AB, Zicari M, Zecchini Antoniolli S, et al. Vesicouterine fistulas following cesarean section: report on a case, review and update of the literature. Int Urol Nephrol 2002;34:335-44.

5. Al Nuaim LA, Kattan S, Mustafa MS. Vesicouterine fistula after a previous low vertical cesarean section (Delee incision). Int J Gynaecol Obstet 1996:55:161-2.

6. Novi JM, Rose M, Shaunik A, et al. Conservative management of vesicouterine fistula after uterine rupture. Int Urogynecol J Pelvic Floor Dysfunct 2004; 15:434-5.

7. Jozwik M, Jozwik M, Lotocki W. Vesicouterine fistula-an analysis of 24 cases from Poland. Int J Gynaecol Obstet 1998:57:169-72.
8. Sefrioui 0 , Benabbes Taari H, Azyez M, et al. Vesico-uterine fistula of obstetrical origin. Report of 3 cases [article in French]. Ann Urol (Paris) 2002:36:376-80.

9. Alkatib M, Franco AV, Fynes MM. Vesicouterine fistula following Cesarean delivery-ultrasound diagnosis and surgical management. Ultrasound Obstet Gynecol 2005;26:183-5.

10. Tarhan F, Erbay E, Penbegul N, et al. Minimal invasive treatment of vesicouterine fistula: a case report. Int Urol Nephrol 2007:39:791-3.

11. Jozwik M, Jozwik M. Spontaneous closure of vesicouterine fistula. Account for effective hormonal treatment. Urol Int 1999:62:183-7.

12. Eogan M, McKenna P. Conservative management of a traumatic uterovesical fistula ('Youssef's syndrome'). Eur J Obstet Gynecol Reprod Biol 2003;110:114-6.

13. Ramalingam $M$, Senthil K, Pai $M$, et al. Laparoscopic repair of vesicouterine fistula-a case report. Int Urogynecol J Pelvic Floor Dysfunct 2008;19:731-3.

14. Waaldijk K. The immediate management of fresh obstetric fistulas. Am J Obstet Gynecol 2004; 191:795-9.

Correspondence: Dr. Mathieu Bettez, Division of Urology, University of Sherbrooke, 3001, 12e Ave Nord, Sherbrooke, QC, JIH 5N4; fax: 819-820-6411; mathieu.bettez@usherbrooke.co 